\title{
A New Perspective on Daily Value at Risk Estimates
}

\author{
Arthur L. Dryver \\ Graduate School of Business Administration, NIDA, Bangkok, Thailand \\ Tel: 66-86-999-8377 E-mail: dryver@gmail.com \\ Sarayut Nathaphan \\ Thammasat Business School, Thammasat University, Bangkok, Thailand
}

\author{
Received: February 6, $2012 \quad$ Accepted: February 22, $2012 \quad$ Published: April 1, 2012 \\ doi:10.5539/ijef.v4n4p114 \\ URL: http://dx.doi.org/10.5539/ijef.v4n4p114
}

The Graduate School of Business Administration, NIDA, Bangkok, Thailand is providing support for this research.

\begin{abstract}
Daily value at risk (VaR) estimates are sometimes calculated as if the institution is only concerned about short-term performance or risk position. In reality though, a risk manager may not consider changing the investment allocation in the foreseeable future, and with a highly-leveraged position daily VaR could be very misleading in terms of true risk to the financial institution. This paper recommends looking at VaR, taking the possibility that a financial institution will use the same assest allocation over a longer period of time while borrowing at over night rates. Finally, the paper introduces a more conservative estimate than the traditional VaR estimates.
\end{abstract}

Keywords: Bonferoni, Risk management, Value at risk

\section{Introduction}

Value at Risk (VaR) is used by risk managers to estimate the potential downside regarding a given time frame for a given confidence level (Jorion, 2007). There are many approaches to estimate VaR; e.g., using different probability distribution functions such as normal, pareto, Cauchy distributions, mixture distributions, etc. (e.g. Ruppert, 2004; Jorion, 2007; Haas, 2009; Dimitrakopoulos et al., 2010; Chin, 2008). These various ways to estimate VaR can yield widely different results (Beder, 1995; Pritsker, 1997, etc.). One of the shortcomings of the VaR measurement for accessing risk is that it does not yield insight into loss beyond the set VaR level $\alpha$ (Yamai and Yoshiba, 2005). Thus the amount one could lose when the portfolio value goes below the threshold at the significant level is unknown. From this perspective, VaR is a conservative measurement of the true amount that could be lost. In addition, as Dankelsson (2002) noted, data collected during good times do not yield much insight into what will occur when times are bad. Some people go as far as to say that VaR should not be used, as it is too misleading (Szegö, 2005). Regardless, it is very important to understand VaR better, as an excerpt from The Financial Crisis Inquiry Commision (2011) illustrates its importance.

"For example, as of 2007, the five major investment banks - Bear Stearns, Goldman Sachs, Lehman Brothers, Merrill Lynch, and Morgan Stanley - were operating with extraordinarily thin capital. By one measure, their leverage ratios were as high as 40 to 1 , meaning for every $\$ 40$ in assets, there was only $\$ 1$ in capital to cover losses. Less than a 3\% drop in asset values could wipe out a firm."

Given the limitations of $\mathrm{VaR}$ and the amount of risk various financial institutions may take on it is important to have more risk management tools. This paper focuses on daily $\mathrm{VaR}$ and introduces a new perspective, leading to a supplemental estimate of the traditional daily VaR estimates to aid in risk management.

\section{Value at Risk Technical Notation}

The 24-hour, daily, $\mathrm{VaR}$ at $\alpha$ means that there is only an $\alpha$ probability of a loss of such a magnitude or worse within a 24-hour time frame (Chan and Wong, 2006). Let $R_{t}$ be defined as the daily return for day $t$ and

$$
R_{t}=\frac{\left(P_{t}-P_{t-1}\right)}{P_{t}}
$$


where $P_{t}$ is the price for day $t$

$P_{t-1}$ is the price for the previous trading day.

Let $W_{0}$ represent the initial investment and let $R^{\alpha}$ be the daily return at the $\alpha \times 100$ percentile. For example, $R^{0.05}$ is the return at the 5 percentile for a day. Thus $R^{\alpha}$ equals the return that yields the daily VaR for $\alpha$ given an initial investment of $W_{0}$. That is

$$
\operatorname{VaR}(\alpha)=-W_{0} R^{\alpha}
$$

From equation 2 it can be seen that the random component in the estimation of $\mathrm{VaR}$ is $R^{\alpha}$, as $W_{0}$ is set by the financial institution. Although value at risk is a widely-used tool to estimate the amount of capital at risk over a given time period with confidence $100 \times(1-\alpha) \%$, unfortunately there are many ways in which to estimate $R^{\alpha}$ (Chan and Wong, 2006; Jorion, 2007). In the following subsections, 2.1 and 2.2, the authors cover 2 common methods for estimating $R^{\alpha}$. For both approaches, let $\left(R_{1}, \ldots, R_{n}\right)$ be independently, identically-distributed real random variables, with the common but unknown cumulative distribution function (cdf) $F(R)$.

\subsection{Empirical Estimation of VaR}

The empirical approach for estimating VaR uses the empirical distribution of the daily returns over time period $n$ days. The empirical (cdf) is defined as

$$
\hat{F}_{n}=\frac{1}{n} \sum_{i=1}^{n} I\left(R_{i} \leq r\right)
$$

where

$$
I\left(R_{i} \leq r\right)=\left\{\begin{array}{l}
1 \text { if } R_{i} \leq r \\
0 \text { if } R_{i}>r
\end{array}\right.
$$

Assuming sufficient data, then the empirical distribution is assumed to be similar to the underlying cdf, as the empirical distribution asymptotically goes to the true cumulative distribution function,

$$
\lim _{n \rightarrow \infty} \hat{F}(r)_{n} \rightarrow F(r)
$$

Finally, the empirical distribution is used to estimate $R^{\alpha}$.

\subsection{Normal Distribution Assumption for Estimation of VaR}

Some researchers estimate $\mathrm{VaR}$, assuming that the returns are normally distributed with a mean of $\mu$ and a standard deviation of $\sigma$, i.e. $R_{t}: N(\mu, \sigma)$. The population mean and standard deviation are typically estimated by the sample mean,

$$
\bar{R}=\frac{1}{n} \sum_{i=1}^{n} R_{i}
$$

nd sample standard deviation,

$$
S_{R}=\sqrt{\frac{1}{(n-1)} \sum_{i=1}^{n}\left(R_{i}-\bar{R}\right)^{2}} .
$$

Then the estimate of the value at risk is (Chan and Wong, 2006)

$$
\operatorname{VaR}(\alpha)=-W_{0} \times\left(\bar{R}+Z_{\alpha} S_{R}\right)
$$

where $Z_{\alpha}$ is the $\alpha$ percentile from the standardized normal distribution, $Z \sim N(0,1)$.

\section{The Real Issue With Using Daily or Other Short Time Frame Var Estimates}

Value at risk is used in part to determine capital requirements for financial institutions (Berkowitz and O'Brien, 2002; Jorion 2007). Daily VaR estimates provide the financial institution with an idea of a worst case scenario for the next day. Unfortunately, this can be very misleading, especially from a long-term perspective. For example, a 
$\operatorname{VaR}(0.05)$ is expected to occur or worse once in 20 days, approximately once a month. To state that a daily $\operatorname{VaR}(0.05)$ has only a $5 \%$ chance of occurring on any one day is very misleading when one will perform the event multiple times, say more than 20 times. In quantitative research researchers perform hypothesis tests and they may set $\alpha$ to be 0.05 for the level of significance, but it is understood that multiple hypothesis tests do not exhibit an overall level of significance of $\alpha$ at 0.05 . The latter reason is why one-way ANOVA is used to test the equality of population means over multiple, two-sample t-tests. If there are 6 factors to test if the population means are equal, then that would equal 15 hypothesis tests and the probability of making an error in at least one of the 15 hypothesis tests and rejecting it when the null is actually true is $1-0.95^{15}=0.5367$, whereas with one-way ANOVA the overall significance level of stating a single factor differs from the other factors is still 0.05 . Unfortunately, daily VaR estimates suffer from a shortcoming similar to multiple two-sample t-tests in quantitative research. It is true for that one day a $\operatorname{VaR}(0.05)$ may have only a $5 \%$ chance of occuring, just as with the probability of a type I error for each of the two-sample tests when considered individually; however, should they be considered individually, we know t-tests are not thus the reason for ANOVA. Now turning back to daily VaR, should daily VaR be treated and looked at as if it were to be performed only once or does an adjustment need to be made?

Let us assume the market is weak form efficient and that each day is independent of the other days (Jones, 2007). Under the latter assumption, then, the probability of $\operatorname{VaR}(\alpha)$ occurring on any given day follows a Bernoulli distribution, with a probability of success $\alpha$. Let $X$ be a random variable equaling the number of times a daily $\operatorname{VaR}(\alpha)$ is observed. When looking at a time frame of $n$ days, then $X$ follows a Binomial distribution $X \sim \operatorname{Bin}(n, \alpha)$. Given the latter assumptions, Tables 1 and 2 were created.

Looking at Table 1, there is a probability of $\operatorname{VaR}(0.01)$ occurring 0.9205545483 times within a year. In fact, we expect it to happen 2.52 times per year, as can be seen in Table 2. For $\operatorname{VaR}(0.0001)$, a risk management advisor could say that there is a one in ten thousand chance of it occurring on a given day. Given this viewpoint, he tells his client or financial regulators not to worry. What if the investor thinks in the long term, say ten years hence? Then there is a 0.2227650558 probability of the VaR occuring on any one day within the ten years, Table 1 . What if this event could cause severe damage to the investor's portfolio given his or her amount of leverage. In addition, this one in ten thousand event and the amount of damage it could cause are highly dependent upon the distribution assumed (see Table 3). As $\alpha$ approaches 0 , the estimate of VaR becomes more dependent on the distribution chosen to estimate it; as an example the Standard Normal distribution is compared to the t-distribution, with 10 degrees of freedom (Table 3).

\section{The New Approach for Daily Var}

This new approach differs according to the way in which VaR is calculated. The authors estimate the daily VaR $\alpha=0.05$ and 0.01 occurring once within 5 and 10 days, basically 1 and 2 work weeks, respectively. In essence, the value at risk for any given day within a given time frame, here being 1 and 2 weeks. The authors propose three methods for estimating the daily value at risk within a given time frame.

1. A Bonferoni type estimation (Neter et al., 1996) and using an assumed distribution. With this method we divide the desired $\alpha$ by the time frame, and here the authors divided $\alpha$ by 5 and 10 for 1 and 2 weeks, respectively. Next we assume a distribution of the underlying stocks, or funds, and estimate their parameters. Finally, we calculate the daily VaR but using the adjusted $\alpha$.

2. A Bonferoni type estimation and using simulation techniques. According to this method, we divide the desired $\alpha$ by the time frame, and in this paper the authors divided $\alpha$ by 5 and 10 for 1 and 2 weeks, respectively. Next we use simulation to create an adequate sample size from the previous performance. Finally, we calculate the daily VaR but using the adjusted $\alpha$.

3. Pure simulation techniques. For this paper we investigated VaR for 1 and 2 weeks time frame. The authors used simulation techniques to create a large population of returns. Then the population of returns was divided into sets of 5 for a week estimate and into sets of 10 for a 2-week estimate. The minimum of each set was taken to represent the worst day of the set. Then the unadjusted $\alpha$ percentiles were determined.

Major indices were used to investigate the VaR under the different approaches. Data were downloaded from Yahoo! Finance and the years studied were 2002 through 2011. The prices used for the calculation were the adjusted closing prices for the indices. There were approximately 2,500 returns for each index. Unfortunately, all of these approaches assume weak form market efficiency. For this reason a runs test was performed to investigate the assumption of weak form efficiency in subsection 4.3.

\subsection{Under Normal Distribution}

In Table 5, method 1 was investigated under the Normal Distribution. 


\subsection{Simulation Results}

Samples of size 200,000 were taken for the simulations. The samples of returns were generated using simple random sampling with replacement.

\subsection{Investigating Market Efficiency and Runs Test}

The weak form efficiency assumption is used in Section 3 to discuss one of the shortcomings of VaR as is. In this subsection, we investigate weak form market efficiency. One test used for testing if the market is of weak form efficiency is the runs test (McInish and Puglisi, 1982; Ojah and Karemera, 1999; Dickinson and Muragu, 1994, etc.). This test checks for streaks of positive or negative trading days. Streaks would imply a lack of independence. The assumption of independence is one of the assumptions in our new approach to $\mathrm{VaR}$, as independence is needed to assume a Binomial distribution.

For the runs test let the expected number of runs be defined as $m$,

$$
m=\frac{N(N+1)-\sum_{i=1}^{3} n_{i}^{2}}{N},
$$

where $N$ is the number of price changes and $n_{1}, n_{2}, n_{3}$ are the numbers of positive price changes, negative price changes, and number of no price changes, respectively. The standard deviation, $\sigma_{m}$, of the number of runs

$$
\sigma_{m}=\left(\frac{\sum_{i=1}^{3} n_{i}^{2}\left[\sum_{i=1}^{3} n_{i}^{2}+N(N+1)\right]-2 N \sum_{i=1}^{3} n_{i}^{3}-N^{3}}{N^{2}(N-1)}\right)^{1 / 2} .
$$

The standardized variable

$$
V=\frac{r+1 / 2-m}{\sigma_{m}}
$$

has an approximately normal distribution, where $r$ is the actual number of runs, and $1 / 2$ is the continuity correction.

\section{Conclusion}

Often we may think of risk in the short term, basically for this case what could I loose today, daily VaR, but when repeating the process over and over again for years, even nightmare scenarios should be expected. According to the simulation results in Tables 5, 6, and 7 that when one repeats the same asset allocation for a week to two weeks and pretends it is only for a day one can underestimate the VaR by approximately $23 \%$ to $132 \%$. When assuming the Normal Distribution for determining VaR yielded very different and much smaller VaR results than with the empirical distribution. As can be seen in Table 5, using a Normal Distribution assumption, method 1, would lead one to believe that the VaR is underestimated by approximately $23 \%$ to $57 \%$. The simulation results of methods 2 and 3 in Tables 6 and 7 yielded similar results. Many index results estimate that the VaR would be $100 \%$ larger than estimated treating it as daily when the process was repeated over two weeks.

When dealing with multiple hypothesis tests in quantitative research, researchers should and often do make adjustments to keep the overall type 1 error rate at the desired level. A similar example is the fact that researchers will use one-way ANOVA tests over multiple t-tests. VaR is not a hypothesis test, but it uses probabilities in a similar fashion. Unfortunately, given systemic risk, these nightmare scenarios and their probabilities are most likely understated, even in this paper.

Another issue is the possibility of these events happening back to back, two days in a row. That is two instances of observing a $\operatorname{VaR}(0.05)$ one right after the other. This paper assumes a weak form efficient market, but as many state, the market is inefficient, and thus one can expect streaks if this is so (McInish and Puglisi, 1982; Ojah and Karemera, 1999; Dickinson and Muragu, 1994) which could be devastating to a portfolio. Thus the authors recommend that money managers consider this new perspective on VaR and should calculate it along with the traditional VaR estimates for a better perspective on overall risk and VaR.

\section{Acknowledgments}

The authors would like to thank the Graduate School of Business Administration, NIDA for support of this research. 


\section{References}

Beder, T. (1995). VAR: Seductive but dangerous. Financial Analysts Journal, 51(5):12-24. http://dx.doi.org/10.2469/faj.v51.n5.1932

Berkowitz, J., \& O’Brien, J. (2002). How accurate are value-at-risk models at commercial banks? The Journal of Finance, 57(3):1093-1111. http://dx.doi.org/10.1111/1540-6261.00455

Chan, N., \& Wong, H. (2006). Simulation techniques in financial risk management. John Wiley and Sons. http://dx.doi.org/10.1002/0471789496

Chin, W. (2008). Heavy-tailed value-at-risk analysis for Malaysian stock exchange. Physica A: Statistical Mechanics and its Applications, 387(16-17):4285-4298.

Danielsson, J. (2002). The emperor has no clothes: Limits to risk modelling. Journal of Banking \& Finance, 26(7):1273-1296. http://dx.doi.org/10.1016/S0378-4266(02)00263-7

Dickinson, J., \& Muragu, K. (1994). Market efficiency in developing countries: A case study of the Nairobi stock exchange. Journal of Business Finance \& Accounting, 21(1):133-150. http://dx.doi.org/10.1111/j.1468-5957.1994.tb00309.x

Dimitrakopoulos, D., Kavussanos, M., \& Spyrou, S. (2010). Value at Risk Models for Volatile Emerging Markets Equity Portfolios. The Quarterly Review of Economics and Finance.

Haas, M. (2009). Value-at-Risk via mixture distributions reconsidered. Applied Mathematics and Computation, 215(6):2103-2119. http://dx.doi.org/10.1016/j.amc.2009.08.005

Jones, C. (2007). Investments: analysis and management. Wiley-India.

Jorion, P. (2007). Value at risk: the new benchmark for managing financial risk. McGraw-Hill Companies.

McInish, T., \& Puglisi, D. (1982). Efficiency of the International Money Markets. J. Business Finance \& Account, 9(2):167-178. http://dx.doi.org/10.1111/j.1468-5957.1982.tb00983.x

Neter, J., Wasserman, W., Kutner, M., \& Li, W. (1996). Applied linear statistical models. Number L. Irwin.

Ojah, K., \& Karemera, D. (1999). Random walks and market efficiency tests of Latin American emerging equity markets: a revisit. Financial Review, 34(2):57-72. http://dx.doi.org/10.1111/j.1540-6288.1999.tb00454.x

Pritsker, M. (1997). Evaluating value at risk methodologies: accuracy versus computational time. Journal of Financial Services Research, 12(2):201-242. http://dx.doi.org/10.1023/A:1007978820465

Ruppert, D. (2004). Statistics and finance: an introduction. Springer Verlag.

Szegö, G. (2005). Measures of risk* 1. European Journal of Operational Research, 163(1):5-19. http://dx.doi.org/10.1016/j.ejor.2003.12.016

THE FINANCIAL CRISIS INQUIRY COMMISSION. (2011). THE FINANCIAL CRISIS INQUIRY REPORT.

Yamai, Y., \& Yoshiba, T. (2005). Value-at-risk versus expected shortfall: A practical perspective. Journal of Banking \& Finance, 29(4):997-1015. http://dx.doi.org/10.1016/j.jbankfin.2004.08.010

Table 1 . The probability of a daily $\operatorname{VaR}(\alpha)$ occurring within the various time horizons

\begin{tabular}{|r|l|l|l|l|l|}
\hline Days & time frame & $\alpha=0.05$ & $\alpha=0.01$ & $\alpha=0.001$ & $\alpha=0.0001$ \\
\hline 1 & single day & 0.0500000000 & 0.0100000000 & 0.0010000000 & 0.0001000000 \\
\hline 5 & a week & 0.2262190625 & 0.0490099501 & 0.0049900100 & 0.0004999000 \\
\hline 10 & two weeks & 0.4012630608 & 0.0956179250 & 0.0099551198 & 0.0009995501 \\
\hline 63 & a quarter & 0.9605009061 & 0.4690944570 & 0.0610861223 & 0.0062805097 \\
\hline 252 & one year & 0.9999975658 & 0.9205545483 & 0.2228532539 & 0.0248863592 \\
\hline 454 & two years & 0.9999999999 & 0.9895677413 & 0.3650615103 & 0.0443870101 \\
\hline 1260 & five years & 1.000000000 & 0.9999968352 & 0.7165247384 & 0.1183907077 \\
\hline 2520 & ten years & 1.000000000 & 1.0000000000 & 0.9196417761 & 0.2227650558 \\
\hline
\end{tabular}


Table 2. The expected number of occurrences

\begin{tabular}{|r|l|r|r|r|r|}
\hline Days & time frame & \multicolumn{1}{|c|}{$\alpha=0.05$} & \multicolumn{1}{|c|}{$\alpha=0.01$} & $\alpha=0.001$ & $\alpha=0.0001$ \\
\hline 1 & single day & 0.05000 & 0.01000 & 0.00100 & 0.00010 \\
\hline 5 & a week & 0.25000 & 0.05000 & 0.00500 & 0.00050 \\
\hline 10 & two weeks & 0.50000 & 0.10000 & 0.01000 & 0.00100 \\
\hline 63 & a quarter & 3.15000 & 0.63000 & 0.06300 & 0.00630 \\
\hline 252 & one year & 12.60000 & 2.52000 & 0.25200 & 0.02520 \\
\hline 454 & two years & 22.70000 & 4.54000 & 0.45400 & 0.04540 \\
\hline 1260 & five years & 63.00000 & 12.60000 & 1.26000 & 0.12600 \\
\hline 2520 & ten years & 126.00000 & 25.20000 & 2.52000 & 0.25200 \\
\hline
\end{tabular}

Table 3. The importance of the correct distribution when investigating VaR for a very small $\alpha$

\begin{tabular}{|l|c|r|r|r|}
\hline Distribution & $\alpha=0.05$ & $\alpha=0.01$ & $\alpha=0.001$ & $\alpha=0.0001$ \\
\hline$z_{\alpha}=$ & -1.644853627 & -2.326347874 & -3.090232306 & -3.719016485 \\
\hline$t_{(10 \alpha)}=$ & -1.812461102 & -2.763769458 & -4.143700493 & -5.693820101 \\
\hline
\end{tabular}

Table 4. Bonferoni first row is back solving - $1-\left(1-\frac{\alpha}{n}\right)^{n}$.

\begin{tabular}{|l|l|l|l|l|l|r|}
\hline Time frame & Daily & Week & 2 Weeks & Daily & Week & 2 Weeks \\
\hline New approach Daily VaR & $5.000 \%$ & $4.901 \%$ & $4.889 \%$ & $1.000 \%$ & $0.996 \%$ & $0.996 \%$ \\
\hline Adjusted Alpha & $5.000 \%$ & $1.000 \%$ & $0.500 \%$ & $1.000 \%$ & $0.200 \%$ & $0.100 \%$ \\
\hline
\end{tabular}

Table 5. The daily VaR at various at 5\% and $1 \%$ level within a one and two-week time frame. Using Bonferoni adjustments and assuming a Normal Distribution, method 1.

\begin{tabular}{|l|l|l|l|l|l|l|l|l|l|l|l|}
\hline On a single Day VaR & \multicolumn{9}{|c|}{$5.000 \%$} & \multicolumn{5}{c|}{$1.000 \%$} \\
\hline & \multicolumn{3}{|c|}{ VaR $W_{0}=1$} & \multicolumn{1}{|c|}{ VaR $\div$ Daily VaR } & \multicolumn{3}{c|}{ VaR $W_{0}=1$} & \multicolumn{2}{c|}{ VaR $\div$ Daily VaR } \\
\hline Time frame & Daily & Week & 2 Weeks & Week & 2 Weeks & Daily & Week & 2 Weeks & Week & 2 Weeks \\
\hline Adjusted Alpha & $5.000 \%$ & $1.000 \%$ & $0.500 \%$ & $1.000 \%$ & $0.500 \%$ & $1.000 \%$ & $0.200 \%$ & $0.100 \%$ & $0.200 \%$ & $0.100 \%$ \\
\hline S and P 500 & 0.0227 & 0.0321 & 0.0356 & 1.4167 & 1.5692 & 0.0321 & 0.0398 & 0.0427 & 1.2382 & 1.3297 \\
\hline Dow & 0.0210 & 0.0298 & 0.0330 & 1.4175 & 1.5703 & 0.0298 & 0.0369 & 0.0397 & 1.2385 & 1.3301 \\
\hline Nasdaq & 0.0255 & 0.0361 & 0.0400 & 1.4181 & 1.5711 & 0.0361 & 0.0447 & 0.0481 & 1.2387 & 1.3305 \\
\hline FTSE & 0.0218 & 0.0309 & 0.0343 & 1.4165 & 1.5690 & 0.0309 & 0.0383 & 0.0411 & 1.2381 & 1.3296 \\
\hline DAX & 0.0269 & 0.0382 & 0.0423 & 1.4172 & 1.5699 & 0.0382 & 0.0473 & 0.0508 & 1.2384 & 1.3300 \\
\hline CAC 40 & 0.0263 & 0.0371 & 0.0411 & 1.4140 & 1.5656 & 0.0371 & 0.0460 & 0.0493 & 1.2371 & 1.3282 \\
\hline NIKKEI 225 & 0.0261 & 0.0369 & 0.0408 & 1.4147 & 1.5665 & 0.0369 & 0.0456 & 0.0490 & 1.2374 & 1.3286 \\
\hline HANG SENG & 0.0263 & 0.0373 & 0.0413 & 1.4194 & 1.5730 & 0.0373 & 0.0462 & 0.0496 & 1.2393 & 1.3312 \\
\hline STRAITS TIMES & 0.0203 & 0.0288 & 0.0319 & 1.4199 & 1.5736 & 0.0288 & 0.0357 & 0.0384 & 1.2394 & 1.3315 \\
\hline
\end{tabular}

Table 6. The daily VaR at various Bonferoni adjusted $\alpha$ levels within a one and two-week time frame, method 2

\begin{tabular}{|l|c|c|r|r|r|r|r|r|r|r|r|}
\hline Daily VaR & \multicolumn{9}{|c|}{$5.000 \%$} & \multicolumn{5}{|c|}{$1.000 \%$} \\
\hline & \multicolumn{3}{|c|}{ VaR $W_{0}=1$} & \multicolumn{1}{|c|}{ VaR $\div$ Daily VaR } & \multicolumn{3}{|c|}{ VaR $W_{0}=1$} & \multicolumn{2}{c|}{ VaR $\div$ Daily VaR } \\
\hline Time frame & Daily & Week & 2 Weeks & Week & 2 Weeks & Daily & Week & 2 Weeks & Week & 2 Weeks \\
\hline S and P 500 & 0.0220 & 0.0415 & 0.0503 & 1.8845 & 2.2805 & 0.0415 & 0.0671 & 0.0881 & 1.6160 & 2.1203 \\
\hline Dow & 0.0200 & 0.0368 & 0.0464 & 1.8382 & 2.3193 & 0.0368 & 0.0582 & 0.0733 & 1.5812 & 1.9940 \\
\hline Nasdaq & 0.0253 & 0.0411 & 0.0507 & 1.6237 & 2.0054 & 0.0411 & 0.0580 & 0.0847 & 1.4131 & 2.0630 \\
\hline FTSE & 0.0215 & 0.0397 & 0.0497 & 1.8430 & 2.3072 & 0.0397 & 0.0544 & 0.0716 & 1.3700 & 1.8045 \\
\hline DAX & 0.0260 & 0.0496 & 0.0536 & 1.9115 & 2.0651 & 0.0496 & 0.0649 & 0.0701 & 1.3086 & 1.4134 \\
\hline CAC 40 & 0.0255 & 0.0460 & 0.0540 & 1.8063 & 2.1205 & 0.0460 & 0.0631 & 0.0683 & 1.3712 & 1.4859 \\
\hline NIKKEI 225 & 0.0241 & 0.0425 & 0.0556 & 1.7617 & 2.3030 & 0.0425 & 0.0938 & 0.0962 & 2.2061 & 2.2629 \\
\hline HANG SENG & 0.0246 & 0.0477 & 0.0518 & 1.9403 & 2.1072 & 0.0477 & 0.0719 & 0.0830 & 1.5065 & 1.7390 \\
\hline STRAITS TIMES & 0.0193 & 0.0335 & 0.0385 & 1.7323 & 1.9950 & 0.0335 & 0.0525 & 0.0734 & 1.5697 & 2.1938 \\
\hline
\end{tabular}


Table 7. The daily VaR at the $5 \%$ and $1 \%$ level within a one and two-week time frame. The calculations in this table uses the minimum for a single day within $n$ days, 1,5 (week), or 10 (two weeks), method 3.

\begin{tabular}{|c|c|c|c|c|c|c|c|c|c|c|}
\hline \multirow{3}{*}{$\begin{array}{l}\text { On a single Day VaR } \\
\text { Time frame }\end{array}$} & \multicolumn{5}{|c|}{$5.000 \%$} & \multicolumn{5}{|c|}{$1.000 \%$} \\
\hline & \multicolumn{3}{|c|}{$\operatorname{VaR} W_{0}=1$} & \multicolumn{2}{|c|}{$\mathrm{VaR} \div$ Daily $\mathrm{VaR}$} & \multicolumn{3}{|c|}{$\operatorname{VaR} W_{0}=1$} & \multicolumn{2}{|c|}{$\mathrm{VaR} \div$ Daily VaR } \\
\hline & Daily & Week & 2 Weeks & Week & 2 Weeks & Daily & Week & 2 Weeks & Week & 2 Weeks \\
\hline $\mathrm{S}$ and $\mathrm{P} 500$ & 0.0220 & 0.0403 & 0.0503 & 1.8280 & 2.2805 & 0.0415 & 0.0671 & 0.0881 & 1.6160 & 2.1203 \\
\hline Dow & 0.0200 & 0.0368 & 0.0464 & 1.8382 & 2.3193 & 0.0368 & 0.0556 & 0.0733 & 1.5130 & 1.9940 \\
\hline Nasdaq & 0.0253 & 0.0409 & 0.0507 & 1.6165 & 2.0054 & 0.0411 & 0.0580 & 0.0847 & 1.4131 & 2.0630 \\
\hline FTSE & 0.0215 & 0.0395 & 0.0497 & 1.8342 & 2.3072 & 0.0397 & 0.0544 & 0.0716 & 1.3700 & 1.8045 \\
\hline DAX & 0.0260 & 0.0496 & 0.0536 & 1.9098 & 2.0651 & 0.0496 & 0.0649 & 0.0701 & 1.3086 & 1.4134 \\
\hline CAC 40 & 0.0255 & 0.0460 & 0.0540 & 1.8063 & 2.1205 & 0.0460 & 0.0631 & 0.0683 & 1.3712 & 1.4859 \\
\hline NIKKEI 225 & 0.0241 & 0.0424 & 0.0556 & 1.7551 & 2.3030 & 0.0425 & 0.0938 & 0.0962 & 2.2061 & 2.2629 \\
\hline HANG SENG & 0.0246 & 0.0477 & 0.0518 & 1.9403 & 2.1070 & 0.0477 & 0.0719 & 0.0830 & 1.5065 & 1.7390 \\
\hline STRAITS TIMES & 0.0193 & 0.0335 & 0.0385 & 1.7323 & 1.9950 & 0.0335 & 0.0525 & 0.0734 & 1.5697 & 2.1938 \\
\hline
\end{tabular}

Table 8. Two-sided Runs Test results

\begin{tabular}{|l|r|r|}
\hline & Z-statistic & p-value \\
\hline S and P 500 & 4.0526 & 0.0001 \\
\hline Dow & 3.2937 & 0.0010 \\
\hline Nasdaq & 0.5869 & 0.5573 \\
\hline FTSE & 2.2351 & 0.0254 \\
\hline DAX & 1.3404 & 0.1801 \\
\hline CAC 40 & 2.6594 & 0.0078 \\
\hline NIKKEI 225 & 2.3509 & 0.0187 \\
\hline HANG SENG & 0.5002 & 0.6170 \\
\hline STRAITS TIMES & 1.8807 & 0.0600 \\
\hline
\end{tabular}

